\title{
Detection of small targets in maritime conditions
}

Stanisław Milewski

\author{
${ }^{*}$ Akademia Marynarki Wojennej , Poland
}

\begin{abstract}
The aim of this article is to discuss some important issue which are relevant to search and detection of targets in maritime conditions. This paper consist of detection theory, thermal experiences and some examples of maritime objects which are examined in aspect to its detection in infrared. Simulation of small surface targets detection was carried out with the Matlab application.
\end{abstract}

\section{Introduction}

Detection of the surface targets is the final task of the search process. However, in maritime condition, the horizon line must be pointed in the thermal image in advance. First of all, the horizon line makes possible to distinguish two different backgrounds in the thermal scene, such as sea (water) and air. In more advanced applications the horizon line enables observers to stabilize the field of view and predict the detection range of searching targets.

Ship detection on the horizon is similar to detection of small object in heterogeneous background. Therefore, the search process and next ship detection is analyzed as the track and detection of the changes of background radiant intensity. The detection model is based on the image signal-to-noise rations (SNRs), which is defined in terms of range and time of observation.

In the target detection models, especially in Automatic Target Recognition (ATR), the first task is to calculate statistic radiant intensity, which is considered as a background in the observed scene. This value is also considered to determine some threshold value of $S N R_{\text {th }}$ and what is more this threshold value is changing in time.

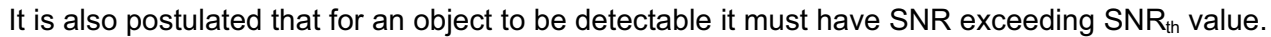

\section{Maritime conditions and target detection process}

There are few examples of the methods, which are used to determine the horizon line, in literature. This problem is not popular because probably small number of people is interested in this terms or there are other unknown causes. The method, I use in my work is presented in figure 1.

The location of the horizon is characterized by examine of the pixel intensity in vertical line in an image ( in other words in line astern of matrix of image).

The horizon in a thermal image (raw line or raw vector in matrix) is described by the line which connects pixels for which in its neighbourhood appears the biggest changes of radiant intensity. This pixels' neighbourhood consists of two background: water and air. Therefore, in maritime conditions, very often the horizon (optical horizon) must be consider and analyze as a few line of an image. The sensibility of the intensity changes is fixed on the basis of experience.

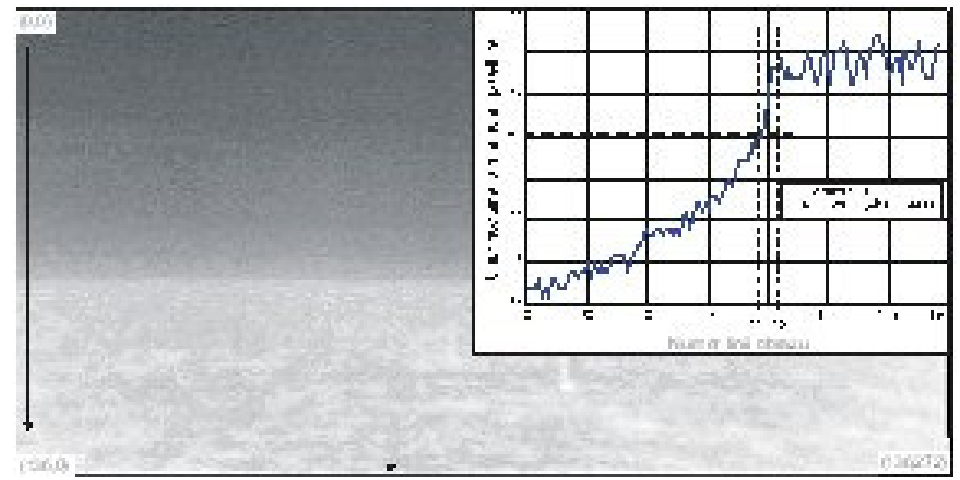

Fig. 1. This figure shows the selected method used to determine of the horizon line in a thermal image

\section{Search and target detection}

Simulation of detection of small surface targets was carried out with the Matlab. With this language the application called "Sygnater" was worked out as a interface for examine both maritime conditions and model of targets detection. Moreover, this application may be used as a database for collection of thermal signatures of the targets.

For known targets the detection model was worked out on the basis of procedure which finally may be described as 


$$
10 \log \left(\frac{\operatorname{SNR}\left(I_{T}\right)}{\operatorname{SNR}\left(I_{B}\right)}\right) \geq X
$$

where:

$X$ - the value which fallows experiment;

SNR (IT) - signal to noise ratio for images with target;

SNR (IB) - signal to noise ratio for images without target.

\section{Results of target detection}

Examples of results of target search and detection for fixed characteristics of maritime conditions and objects are shown in figure 2. For the detection model described by the pattern above, detection process was divided into 3 basic periods of observation, accordance with time: T1,T2,T3.

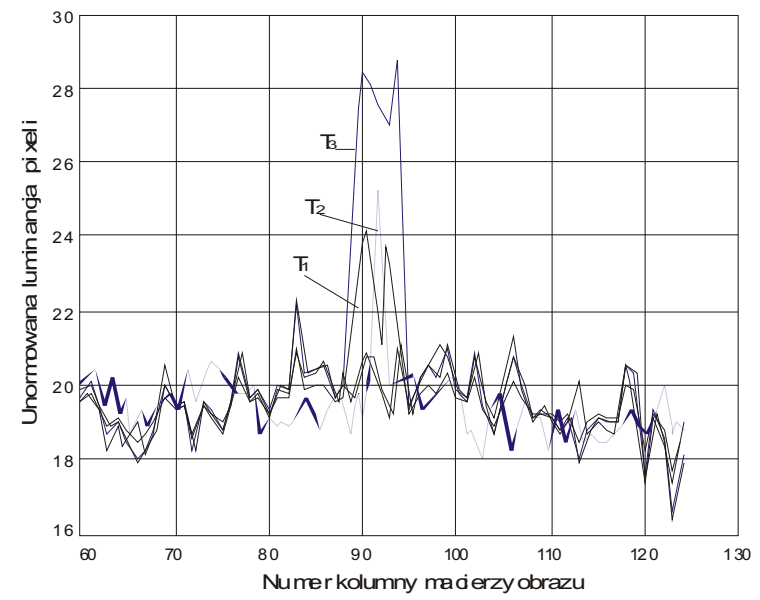

Fig. 2. Detection of suitably number of pixels belong to target in terms of time observation

It is worth mentioning that the detection process is analyzed in the term (1), however it depends on number of suitably pixels for which the SNRTH term is fulfilled. For example, according to O'Nile's criterion, to state that a ship in maritime conditions is detected it requires to distinguish 36 pixels. 